\title{
The Mental Health State of Quiescent Inflammatory Bowel Disease Patients
}

\author{
Sung Chul Park* and Yoon Tae Jeen ${ }^{\dagger}$ \\ "Division of Gastroenterology and Hepatology, Department of Internal Medicine, Kangwon National University School of Medicine, \\ Chuncheon, and ${ }^{\dagger}$ Division of Gastroenterology and Hepatology, Department of Internal Medicine, Korea University Anam Hospital, Korea \\ University College of Medicine, Seoul, Korea
}

See "Factors Associated with Anxiety and Depression in Korean Patients with Inactive Inflammatory Bowel Disease" by Min Chul Kim, et al. on page 399-405, Vol. 10. No. 3, 2016

\begin{abstract}
Although some controversies exist, it is well known that psychological disorders are prevalent in patients with inflammatory bowel diseases (IBD). Previous studies have shown that, as with any patients with chronic physical illness, many IBD patients (21\% to $35 \%$ ) present with anxiety and depression. ${ }^{1}$ During the relapses, it has been reported that more than $80 \%$ of patients showed anxiety state and 60\% showed depression. ${ }^{2}$
\end{abstract}

It has yet to be proven what kind of cause-and-effect relationship exists between IBD and anxiety and depression. These unstable emotional states of IBD patients can aggravate pain and gastrointestinal symptoms by increasing inflammatory activity and lower the patients' quality of life by contributing to feelings of fatigue and decreasing the motivation needed to overcome the disease. ${ }^{3}$ Therefore, it is important to evaluate the prevalence of anxiety and depression in patients with IBD and to implement an appropriate treatment plan. In previous studies, the factors reported to affect the mood disorders included gender, age, education, socioeconomic deprivation, knowledge score, disease duration, extraintestinal manifestations such as arthritis and stomatitis, use of steroid or immunosuppressants, treatment adherence, disease activity, previous bowel surgery, distribution of inflammation, and disease related quality of life. ${ }^{4-6}$ However, in Asian countries like Korea, not much research has been conducted on such topics. A recently published study reported that anxiety and depression is common in Korean IBD patients in remission and suggested that appropriate management be provided for these patients. ${ }^{7}$

In this issue of Gut and Liver, Kim et al. ${ }^{8}$ evaluated the prevalence of anxiety and depression in Korean inactive IBD patients and investigated the predictive factors related with disease and sociodemographic status. A total of 142 IBD patients (75 with ulcerative colitis [UC] and 67 with Crohn's disease [CD]) completed a self-report questionnaire including the Hospital Anxiety and Depression Score (HADS). Because HADS is less influenced by topics related to physical illnesses such as fatigue and wellbeing, it is commonly used to screen mental disorders in IBD patients. Within CD patients, 44\% were anxious and 15\% were depressed, and within UC patients, 41\% were anxious and 24\% were depressed. Although there were no healthy matched controls, these prevalence rates were greater than those of the general population in Korea.

Some studies found that the amount of psychological distress in IBD patients were associated with disease activity, and the prevalence of anxiety and depression in quiescent IBD patients did not differ significantly from that of the general population. ${ }^{2,9,10}$ However, irritable bowel syndrome-like symptoms was two to three times more frequent in quiescent IBD patients than in the control group, and this had consistent associations with anxiety and depression. ${ }^{10}$ In a recent study on Korean inactive IBD patients, the prevalences of anxiety and depression were $27.4 \%$ and 33.6\%, respectively, higher than the 26\% and 9\% previously reported by the Western study using the HADS. ${ }^{7,10}$ Especially within the CD patients, results showed higher frequency for mood disorder compared to the healthy controls. In this study by Kim et al., ${ }^{8}$ the prevalence of anxiety and depression was reported to be high in both quiescent CD and UC patients, suggesting that a significant number of the Korean inactive IBD patients have mood disorder.

The reason for the high prevalence of mood disorders in this study may be that the results were affected by the method used

Correspondence to: Yoon Tae Jeen

Department of Internal Medicine, Korea University Anam Hospital, Korea University College of Medicine, 73 Inchon-ro, Seongbuk-gu, Seoul 02841, Korea

Tel: +82-2-920-6555, Fax: +82-2-953-1943, E-mail: ytjeen@korea.ac.kr pISSN 1976-2283 eISSN 2005-1212 http://dx.doi.org/10.5009/gnl16034

@) This is an Open Access article distributed under the terms of the Creative Commons Attribution Non-Commercial License (http://creativecommons.org/licenses/by-nc/4.0) which permits unrestricted non-commercial use, distribution, and reproduction in any medium, provided the original work is properly cited. 
to recruit the subjects. In this study, the interviews were carried out face to face when the patients visited the hospital, but in most previous studies, excluding that conducted in Korea, the questionnaires were sent by the mail. ${ }^{7}$ The location and time in which the questionnaire was conducted could have affected the patients' comprehension and participation of the questionnaires, and mood disorders may be more common in patients who visited the hospital than in those who participated through the mail. ${ }^{7}$ Therefore, there are some limitations in comparing the prevalence of this study with the Western studies.

In this study, the factors associated with anxiety or depression in CD patients were socioeconomic deprivation and disease duration. No significant independent predictor existed in UC patients, but low income tended to show an association with depression $(p=0.096)$. Socioeconomic deprivation was assessed using the Evaluation of Precarity and Inequalities in Health Examination Centers score called EPICES, which consisted of topics like marriage, health insurance status, economic status, family support, and leisure activities. Another recent study evaluating the risk factors of depression in Korean CD patients reported that subjects who graduated from high school or college and had a low economic status and those with healthrelated quality of life scores lower than 50 points were considered to be at significant risk of developing depression. ${ }^{4}$ Even after IBD went remission, there were many cases of relapse and the consistent hospital care and treatment may lead to difficulties in carrying out patients' school and work lives. When these patients then experience social isolation and difficult economic status, they may show maladaptive coping strategies, potentially increasing the possibility of anxiety and depression. Particularly in the case of $\mathrm{CD}$, relapse is common, and the surgery usually does not lead to cure the disease. Hence, the risk of depression could increase over time for patients with $\mathrm{CD}$ after diagnosis.

A selection bias may exist in this study because only IBD patients who visited a single hospital institution and agreed to participate in the study included. Nonetheless, the results showing that a large proportion of IBD patients, despite being in remission, experience anxiety and depression is an important message. Clinicians should pay attention to the identification and treatment of mood disorder in IBD patients, especially with socioeconomic deprivation and long disease duration. In addition, social support programs may be necessary for the vulnerable IBD patients. To this end, the employment and income of the IBD patients as well as their insurance enrollment rates should be investigated, and the medical expense support of government for the patients should be expanded. In conclusion, clinicians and government agency should attain a deep interest in the mental health and socioeconomic states of IBD patients regardless of disease activity.

\section{CONFLICTS OF INTEREST}

No potential conflict of interest relevant to this article was reported.

\section{REFERENCES}

1. Prasko J, Jelenova D, Mihal V. Psychological aspects and psychotherapy of inflammatory bowel diseases and irritable bowel syndrome in children. Biomed Pap Med Fac Univ Palacky Olomouc Czech Repub 2010;154:307-314.

2. Addolorato G, Capristo E, Stefanini GF, Gasbarrini G. Inflammatory bowel disease: a study of the association between anxiety and depression, physical morbidity, and nutritional status. Scand J Gastroenterol 1997;32:1013-1021.

3. Yoo S, Jung YS, Park JH, et al. Fatigue severity and factors associated with high fatigue levels in Korean patients with inflammatory bowel disease. Gut Liver 2014;8:148-153.

4. Cho OH, Yoo YS, Yang SK. Depression and risk factors in patients with Crohn's disease. J Korean Acad Nurs 2012;42:207-216.

5. Fuller-Thomson E, Sulman J. Depression and inflammatory bowel disease: findings from two nationally representative Canadian surveys. Inflamm Bowel Dis 2006;12:697-707.

6. Nahon S, Lahmek P, Durance C, et al. Risk factors of anxiety and depression in inflammatory bowel disease. Inflamm Bowel Dis 2012;18:2086-2091.

7. Kim ES, Cho KB, Park KS, et al. Predictive factors of impaired quality of life in Korean patients with inactive inflammatory bowel disease: association with functional gastrointestinal disorders and mood disorders. J Clin Gastroenterol 2013;47:e38-e44.

8. Kim MC, Jung YS, Song YS, et al. Factors associated with anxiety and depression in Korean patients with inactive inflammatory bowel disease. Gut Liver 2016;10:399-405.

9. Häuser W, Janke KH, Klump B, Hinz A. Anxiety and depression in patients with inflammatory bowel disease: comparisons with chronic liver disease patients and the general population. Inflamm Bowel Dis 2011;17:621-632.

10. Simrén M, Axelsson J, Gillberg R, Abrahamsson H, Svedlund J, Björnsson ES. Quality of life in inflammatory bowel disease in remission: the impact of IBS-like symptoms and associated psychological factors. Am J Gastroenterol 2002;97:389-396. 